\title{
A transcriptome analysis identifies potential preventive and therapeutic approaches towards COVID-19.
}

Rezaul Islam ${ }^{1, *}, \#$, Andre Fischer ${ }^{1,2,3,{ }^{*}, \#}$

${ }^{1}$ Department for Systems Medicine and Epigenetics, German Center for Neurodegenerative Diseases (DZNE), Von Siebold Str. 3a, 37075, Göttingen, Germany

${ }^{2}$ Department of Psychiatry and Psychotherapy, University Medical Center Göttingen, Germany

${ }^{3}$ Cluster of Excellence "Multiscale Bioimaging: from Molecular Machines to Networks of Excitable Cells"

(MBExC), University of Göttingen, Germany

*Both authors contributed equally to this work.

\# To whom correspondence should be addressed:

Prof. Dr. Andre Fischer; andre.fischer@dzne.de

Dr. Rezaul Islam; mrezaulislam33@gmail.com

\section{Abstract}

The recent outbreak of Coronavirus Disease 2019 (COVID-19) is a major threat to human health and the global economy. In addition to the development of vaccines there is an urgent need for preventive and therapeutic strategies towards severe COVID-19. Key to this would be a better understanding of the molecular mechanisms affected by SARSCOV-2. To address this, we performed a systems biology approach by integrating available RNA-seq datasets from post-mortem lung tissue of COVID-19 patients and cell culture models infected with SARS-COV-2, Respiratory Syncytial virus or influenza virus. We identified two gene-expression modules that are commonly regulated by the three viral diseases and one that is specific to COVID-19. All 3 gene-expression modules represent key inflammatory processes. We identified several proteins within these networks that can be targeted by FDA approved drugs. Key examples are TNF, NFkB, INTERLEUKIN-1 and ALOX5 signaling pathways. Our data also suggest that Vitamin D supplementation and a ketogenic diet should be further analyzed as preventive strategies. In conclusion, our data highlights the potential of transcriptomics to unravel the pathological processes related to COVID-19 and guide the initiation of clinical trials. 


\section{Introduction}

42 Novel coronavirus disease 2019 (COVID-2019) is caused by the severe acute respiratory syndrome coronavirus 2 (SARS-CoV-2). In humans SARS-Cov2 can lead to acute respiratory syndromes eventually causing end stage lung injury, failure of multiple organs and death1. The current SARS-Cov2 pandemic has spread across the globe and has already killed thousands of individuals since the first case was reported in late December, 2019 in Wuhan, China. On 30th January, 2020 WHO declared the outbreak of COVID-2019 a public health emergency of international concern ${ }^{2}$. There are enormous international research efforts to better understand SARS-Cov2 and important findings such as the structural analysis of the Spike (s) protein ${ }^{3}$ by which the virus gains entry to cells or the viral RNA polymerase ${ }^{4}$ have been reported. There is also evidence that SARS-Cov2 recognizes the receptor angiotensin converting enzyme II (ACE2) for cell entry ${ }^{5}$. Interestingly, while most research focuses on the effect of SARS-Cov19 on the general organs such as the lung, a neuro-invasive potential has been observed for SARS-Cov2 and patients often present with neurological symptoms ${ }^{6}$. These data highlight the urgent need to better understand Covid-19 and develop preventive and therapeutic strategies. Several research institutes, biotech and pharma companies are currently developing vaccines against COVID-19. A recent release from WHO (4 ${ }^{\text {th }}$ of April, 2020) listed 63 of such projects ${ }^{7}$ that are however in preclinical or early clinical stages. In addition to vaccination, there is an urgent need for preventive and therapeutic strategies to reduce the rising death toll due to COVID-19. Thus, a better understanding of the molecular pathology associated with SARS-CoV-2 infections is of utmost importance since it also offers the chance for drug repurposing. Some treatment options using several broad-spectrum antivirals such as favipiravir, remdesivir ${ }^{8}$, and anti-malaria drug chloroquine ${ }^{9}$ have been suggested and await further validation. In addition, severe COVID-19 is associated with a major immune inflammatory response and anti-inflammatory therapies are discussed as a promising therapeutic avenue ${ }^{10,11}$.

The analysis of gene-expression networks is a suitable approach to elucidate pathomechanisms and identify drug targets ${ }^{12}$. In this study, we use a systems biology approach to integrate the available transcriptomic data on COVID-19 patients and corresponding cellular models. Our data confirm that COVID-19 infection induces transcriptional changes associated with a major inflammatory response. Further 
analysis revealed that COVID-19 shares a conserved inflammation related gene regulatory network with two other respiratory viruses namely Respiratory Syncytial virus (RSV) and Influenza virus. We also detected a network linked to inflammation that is specific to Covid-19. On the basis of these networks, we provide evidence that anti-inflammatory drugs such as inhibitors of tumor necrosis factor (TNF), NFkB, IL-1 and Alox5 signaling would be bona fide drug targets to treat severe COVID-19. Our data moreover suggest that a ketogenic diet and vitamin $D$ supplementation could be a preventive approach for a severe course of COVID-19.

\section{Results}

\section{Conserved gene-expression networks in COVID-19 are linked to inflammation}

Starting point of our study were RNA-seq datasets obtained from post-mortem lung tissue of COVID-19 patients and corresponding lung-biopsy tissue from uninfected healthy subjects and data from corresponding cell lines. Since data from patients is still rare we decided to start our analysis with more robust data from cell culture models of COVID-19 and related diseases and later cross-correlate these data to findings from patients (Fig. 1A). Our first aim was to identify gene-expression modules that are observed in COVID-19 and related diseases. Thus, we analyzed RNA sequencing data from experiments that employed the same experimental setting using lung epithelial cell line A549 and Calu3 infected with SARS-CoV-2 ${ }^{13}$ and the A549 cell line infected with Respiratory Syncytial Virus (RSV) ${ }^{13}$. As control, we employed lung epithelial cell lines subjected to mock infection. Unbiased weighted gene co-expression (WGCNA) analysis revealed 9 different expression modules in the data (Fig. 1B, Table S1) of which 5 modules displayed high correlation (correlation coefficient $>0.75,{ }^{*} \mathrm{P}<0.05$ ) with the status of viral infection in all experimental conditions (Fig. 1B). While the other four modules would represent cell type or viral infection specific modules, we first decided to further analyze the five modules commonly affected following SARS-CoV2 and RSV infections. Three of these five modules showed decreased expression pattern in both A549 and Calu3 cells while the other two modules displayed increased expression pattern following SARS-CoV-2 infection (Fig. 1C). These changes were consistent in RSV infected cells and data suggest that COVID-19 shares pathological gene-expression changes with related viral diseases. GO analysis of the 3 down- 
108 regulated modules revealed that the MEDarkred module plays a role in mitochondrial 109 function, cellular respiration, and protein folding (Fig. 1D). In line with this, our data 110 suggest that also MEyellow module is linked to cellular respiration and mitochondrial 111 functions (Fig. 1E), while the MEgrey60 module regulates cell cycle check point and 112 genomic repair mechanisms (Fig. 1G). Interestingly, genes of the two up-regulated 113 modules where linked to inflammatory processes (Fig. 1G, 1H). Thus, the MElightcyan 114 module represents biological processes related to increased innate immune response 115 and increased signaling via tumor necrosis factor (TNF), interleukin 1 (IL-1) and NF-kb 116 (Fig. 1G). Genes of the MEmidnightblue module are also involved in immune 117 responses, NF-kb, TNF signaling and viral life cycle (Fig. 1H). In conclusion, the RNA118 seq data analyses suggest that similar inflammatory responses are induced by COVID11919 and Respiratory Syncytial Virus pointing to the possibility that existing anti120 inflammatory therapies may help in the case of severe COVID-19 cases. To further support this hypothesis, we decided to compare our data to findings obtained from an independent study on the influenza virus (InV) that can also cause respiratory syndromes. To this end we employed RNA-seq data generated from lung epithelial A549 cells that were exposed to InV. In line with the original study ${ }^{14}$, we observed a substantial number of deregulated genes (Table S2). Gene enrichment analysis revealed that the up-regulated genes are linked to inflammatory processes including activation of the innate immune response, cellular responses to virus and interferons and activation of T-cells (Fig. 2A). Next, we asked whether the expression changes of

130 two up-regulated modules linked to inflammation (i.e. MElightcyan and 131 MEmidnightblue) that were identified in lung cells treated with COVID-19 are also 132 affected in cells infected with influenza virus. To this end we constructed eigenvalues 133 of the corresponding genes and compared their expression between control and 134 influenza infected samples. Interestingly, the expression of genes within the 135 MElightcyan and MEmidnightblue modules was substantially increased in influenza 136 infected cells (Fig. 2B, 2C). These data further support the view that SARS-COV-2 137 infection drives a conserved gene regulatory network linked to inflammatory processes 138 that is similar to respiratory syndromes caused by RSV and Influenza viruses, pointing 139 to common patho-mechanisms. Moreover, our data provide evidence for the 140 hypothesis that common anti-inflammatory therapies might be a suitable approach 141 towards severe COVID-19. 
143 To substantialize this view we retrieved available RNA-seq dataset on post-mortem

144 lung samples obtained from COVID-19 patients ${ }^{13}$. This dataset also included lung 145 biopsies from uninfected healthy subjects that were processed similarly and used as 146 controls. Although care has to be taken since biopsy and post-mortem data is 147 compared and moreover the data set consist of only 2 patients and 2 controls, a 148 differential expression analysis revealed deregulation of 774 genes (Fig. 3A, Table 149 S3). Gene ontology analysis suggest that the upregulated genes are involved in 150 processes related to inflammation and viral interaction, which is in line with the data 151 obtained from cell culture models (Fig. 3B, also see Fig. 1). Indeed, when we 152 compared the MElightcyan and MEmidnightblue gene-expression modules linked to 153 inflammatory processes, we found both modules to be up-regulated in COVID-19 154 patients (Fig. 3C, 3D).

Transcriptomics point to preventive and therapeutics strategies in COVID-19.

158 The finding that COVID-19 seems to be linked to the up-regulation of 2 conserved gene expression modules related to inflammation allowed us to screen if existing drugs might counteract COVID-19 related pathology. To this end, we constructed the regulatory networks of the 2 modules and asked whether any currently available and FDA approved drugs would target the proteins in these regulatory networks. For MElightcyan module we found a number of proteins (e.g. IFNAR2, XDHH, PTK2B, INSR, JANK2, TNF, ADORA2A, CREB, NFKB1, NFKB2, PTG32) that can be targeted by available drugs. The most important targets are tumor necrosis factor (TNF) and NF-KB1 (Fig. 4A) as they represent the hub genes of the network. This is interesting since various drugs that target these two proteins are available and inhibition of TNF has been recently suggested in a commentary article as a promising strategy to treat severe COVID-1915. In line with these data, chloroquine can inhibit TNF signaling and is already tested in COVID-19 patients ${ }^{9}$. Of particular interest might also be Pranlukast and Thalidomide since they are able to suppress both TNF and NFKB1 signaling and especially Pranlukast has been used to treat asthma in children and adults with no severe side effects ${ }^{16}$. 
175 In the MEmidnightblue module we also find several target proteins that can be 176 modulated by already available drugs (Fig. 4B). For example, immune modulators like 177 IL6, JUN, CD55, TNFS13B, SH2B3, CASP1 and IFNGR2 are potential candidates for 178 which drugs are available and a recent study demonstrated promising results related 179 to COVID-19 management via IL-6 inhibitors ${ }^{17}$. It is also noteworthy that Vitamin D 180 Receptor (VDR) is observed as a potential target within this inflammation regulatory 181 network, supporting recent ideas that Vitamin D supplementation might be a preventive 182 strategy towards severe COVID-19 ${ }^{18}$. Another interesting observation is the presence 183 of CASP1 as it plays a key role in the inflammasome signaling and control for example 184 IL-1ß signaling which might be suppressed by Minocycline ${ }^{19}$. The data described above are based on the 2 identified conserved gene-expression modules. We also analyzed the dataset from human patient material separately. Although care has to be taken when interpreting this limited dataset alone, we observed IL-1ß and TNF as key targets for therapeutic intervention (Fig. 4C).

190 So far, we focused our analysis on gene-expression modules commonly de-regulated in COVID-19 and other viral diseases such as influenza (See Fig. 2). The reverse approach would be to screen for gene-expression changes specific to COVID-19 that are not observed in RSV or influenza. Indeed, we found that out of the 9 originally identified gene-expression modules, the MEbrown module (see Fig 1) was increased in A549 cells treated with COVID-19, while it was decreased in A549 cells treated with RSV or influenza (Fig. 5A). In line with this observation genes of the MEbrown module were increased in postmortem lung tissue of COVID-19 patient (Fig. 5B). GO-analysis of this module indicates that the corresponding genes are linked to inflammation associated processes (e.g. autophagy and Wnt-signaling) and gene-expression control via histone-modifications and non-coding RNA processes (Fig. 5C). When we generated the network of the MEbrown module and asked for potential targets for FDA approved drugs, we identified ALOX5 a promising target (Fig. 5D). ALOX5 codes for the Arachidonate 5-lipoxygenase, a well-known target for inflammatory processes including respiratory diseases ${ }^{20}$. The complete list of potential drugs identified via the approaches described above is listed in supplemental file. 
208 High-fat low-carbohydrate ketogenic diet may reduce COVID-19 related 209 inflammation

211 So far, our data point to a number of interesting FDA approved drugs that could be 212 considered for further testing but also for epidemiological analysis with the aim to 213 identify factors that may lower the risk for a severe course of COVID-19. For example, 214 a number of the identified drugs are used in chronic inflammatory diseases such as 215 rheumatoid arthritis. There is evidence that patient treated with such drugs can have a 216 lower risk for other inflammatory diseases. For example, Alzheimer's disease (AD) is 217 linked to neuroinflammatory processes and there is substantial evidence from 218 epidemiological studies that patients chronically treated with anti-inflammatory drugs 219 for other reasons have a lower risk to develop $A D$ at old age ${ }^{21}$. On these bases we 220 wondered about general environmental factors that could be associated with 221 inflammatory processes and may therefore provide preventive strategies for severe 222 COVID-19. One interesting example is the finding that a ketogenic diet was shown to 223 reduce inflammation. To address this issue we used a dataset from Goldberg et al. ${ }^{22}$. 224 Here, the authors employed a mouse model of influenza infection and performed RNAseq from lung tissue extracted from mice fed ketogenic diet (KD) or normal chow. We re-analyzed this data (Fig. 6A) and confirmed the previously reported reduced inflammatory responses in KD mice (Fig. 6B). Interestingly, while ketogenic diet had no effect on MElightcyan and MEbrown gene-expression modules (Fig. 6C, 6D), this diet reduced the expression of MEmidnitghblue inflammatory module that we found to be increased COVID-19, RSV and InV (Fig. 6E). Moreover, expression of several viral responsive related genes in COVID-19 were downregulated in mice fed a ketogenic diet (Fig. 6F). In sum, these data indicate the potential of ketogenic diet as a preventive approach to affect the risk for a severe course COVID-19. 


\section{Discussion}

242 This study uses available transcriptome data from cell culture models and postmortem 243 material from COVID-19 patients. Although especially the human data is preliminary 244 due to the small sample size, we aimed to overcome this issue by using an integrative approach by combining the various datasets from cell culture models. A robust observation is the finding that COVID-19 leads to the up-regulation of gene-expression modules that all represent key inflammatory processes. Two conserved geneexpression modules are also regulated in response to RSV or influenza while one module was specific to COVID-19. Overall, these findings are in line with the hypothesis that an aberrant inflammatory response plays a role in severe COVID-19 cases $^{10,11}$. Our data allowed us to identify therapeutic targets within these modules and screen for potential FDA approved drugs. As most promising targets we identify processes related to TNF, NFkB, IL-1ß and ALOX5 signaling. These data are in line with recent proposals to test TNF-related therapies in COVID-19 ${ }^{15}$ and also hint to additional approaches that can be tested immediately. For example, our data suggest that Pranlukast which affects TNF and NFKB-signaling ${ }^{23}$ could be a suitable approach to attenuate COVID-19 related inflammation. Equally interesting is Minocycline towards ALOX5, since this pathway was in our dataset specific to COVID-19 and not observed in datasets from RVS or influenza. Additionally, Minocycline can suppress IL-1ß signaling that was also detected as a key process affected by COVID-19. A potential role of $\mathrm{IL}-1 ß$ is also noteworthy, since ongoing clinical trials are rather focusing on IL-6 as therapeutic target (see https://dzif.clinicalsite.org/de/cat/2084\#c2084). A recent study reported that high glucose levels in blood promote the release of inflammatory cytokines following influenza infection. This was mediated in part via TNF receptor-associated factor 6 (TRAF6) mediated activation of interferon regulatory factor-5 (IRF5). In line with these data, we also observed TRAF6 as one of the hub genes in our gene regulatory networks (see Fig. 3A). In addition to TRAF6, the Insulin receptor (INSR) was present in the same conserved network indicating that a similar glucose dependent increased inflammatory mechanism may persist in COVID-19. In line with this hypothesis, patients with high blood glucose levels (e.g. individuals suffering from diabetes) might be more susceptible to develop severe COVID-1924. Another interesting observation is the presence of Vitamin $\mathrm{D}$ receptor as a key target in the COVID-19 related gene-expression network. This data is in line with reports

273 showing that vitamin D supplements exhibit anti-inflammatory effects a by reducing 274 interleukins and NFKB activity ${ }^{25,26}$. Dietary vitamin D supplementation may be a simple, 
275 safe and inexpensive preventive measure to reduce the risk for severe COVID-19, a 276 hypothesis that should be tested in epidemiological studies. In fact, recent reports have 277 already pointed to a potential beneficial effect of Vitamin D in COVID-19 ${ }^{18}$. Moreover, 278 Vitamin E can suppress ALOX5 to limit inflammation ${ }^{27}$, providing further evidence that 279 strategies towards ALOX5 inhibition should be further tested in COVID-19. It would be 280 important to screen for additional strategies that may impact on the course of COVID281 19. As a first approach we analyzed the effect of ketogenic diet and observed that it 282 might have a beneficial effect since in counteracted the expression changes of at least 283 1 of the 3 gene-expression modules we identified as a consequence of COVID-19.

Although the available data on COVID-19 is still limited, our study highlights the power of gene-expression analysis to identify potential preventive strategies and drug targets for repurposing to treat severe COVID-19. Taking into account that at least 657 clinical studies towards COVID-19 are currently ongoing or in preparation (clinincaltrials.gov), we suggest that the continued analysis of available multi-omics data should become an important approach to guide the development and testing of drugs against COVID19.

\section{Methods}

\section{Data acquisition and analysis}

RNAseq data were retrieved from three studies. The NCBI GEO accession of these studies are as follow: GSE147507, GSE136536 and GSE121155. Compressed FASTQ files were downloaded from corresponding SRA accessions using fastq-dump of sratoolkit (version 2.8.0). The quality of the FASTQ files were inspected using FASTQC (version 1.0). Transcriptomic reads were mapped to corresponding genome using STAR (version 2.5.2b). For human and mouse, hg38 and mm10 genome were used for mapping. The mapped files (BAM) were used to enumerate the reads for each gene using featureCounts of Subread (version 1.5.1). Downstream analyses were performed in $\mathrm{R}$ (version 3.6.1). These raw reads were normalized to the library size and differential expression analysis was performed in DESeq2 ${ }^{28}$. Except otherwise mentioned, genes with adjusted $p$ value $<0.05$ were considered as differentially 
309

310

311

312

313

314

315

316

317

318

319

320

321

322

323

324

325

326

327

328

329

330

331

332

333

expressed. Gene ontology analysis was performed using gene ontology (http://geneontology.org/).

\section{Weighted gene co-expression analysis}

Weighted gene co-expression network analysis was performed using (WGCNA) package $^{29}$ in $\mathrm{R}$ (version 1.61). Briefly, after normalizing expression data, gene expression counts were log (base 2) transformed to calculate pair-wise correlations between genes. A signed gene expression network was constructed using soft threshold power of 17 . Modules of co-expressed microRNAs with a minimum module size of 200 with deepsplit 1 was later identified. Similar modules were merged using dissimilarity correlation threshold of 0.25 . Different modules were summarized as network of modular eigengenes, (MEs) which were then correlated with infection status. Pearson correlation of MEs and each of these features was plotted as heat map. Eigenvalues of the modules in in vivo samples were determined after limiting the module genes to be expressed and deregulated therein. Plots are generated using ggplot2 in R. Gene ontology analyses were performed as described above. Bar plots of gene ontology are plotted using GraphPad Prism (version 7.0).

\section{Gene-Drug interactions analysis}

Gene regulatory network was constructed using STRING ( $v$ 11.0) based on the evidence of protein-protein interactions. Network was visualized in Cytoscape (version 3.7.1). Hub genes of the network was identified using CytoHubba. Top 10 Hubba nodes were ranked using degree of interactions and their shortest paths were displayed. The network was extended and integrated to available FDA approved drugs using CyTargetLinker. The DrugBank database (version 4.0) was used for this purpose.

\section{Statistics}

Statistical analysis was performed using either R or GraphPad Prism 7. Statistical test details are given in the figure legends. 


\section{Acknowledgements}

345 Authors thank Maria Goldberg, Dr. Marwah Karim, Sadman Sakib and Chinmoy Saha 346 for their suggestions to the work. This work was supported by funds to AF from the 347 German Center from Neurodegenerative diseases (DZNE) and the Deutsche 348 Forschungsgemeinschaft (DFG, German Research Foundation) under Germany's 349 Excellence Strategy - EXC 2067/1- 390729940

\section{Figure legends}

353 Fig 1. Weighted co-expression analysis. A. Outline of the experimental approach. B. 354 Signed correlation of identified modules to viral infection. A total of 9 modules were identified. 355 Heatmap showing Pearson's correlation and statistical significance. Numbers in parenthesis 356 indicate $p$ value while numbers on top represent the correlation co-efficient. C. Five highly 357 significant clusters (cor $>0.75,{ }^{*} \mathrm{P}<0.05$ ). Gene expression changes of MEDarkred, MEyellow 358 and MEgrey modules are negatively correlated to infection, while the MElightcyan and MEmidnightblue modules are positively correlated. (D-H) Expression of MEdarkred, MEyellow, MEgrey60, MElightcyan and MEmidnightblue across datasets. In vitro data from two cell lines namely A549 and Calu3 were used for COVID-19 infection. For RSV infection, in vitro transcriptomic data from A549 cell line has been used. Effect on gene expression after viral treatment were compared to that treated with mock of the same experiment. Bar plot shows biological processes. $\mathrm{X}$-axis represent $\log 10$ of adjusted $\mathrm{p}$ value. Two-tailed, unpaired t-test; $p$ value is indicated on the figure panel. $N=2-3$ per group.

Fig 2. MElightcyan and MEmidnightblue modules are up-regulated after infections with influenza virus. A. Gene ontology analysis of the influenza viral induced upregulated genes. Boxplots show that the MElightcyan (B) and MEmidnightblue (C) modules are increased in A549 cells treated with influenza viral titers compared to mock treated cells. Unpaired t-test, two-tailed, $\mathrm{N}=3$; each group. 
377 Fig 3. Case vs. control lung RNAseq data links SARS-CoV-2 infection towards 378 inflammation. A. Volcano plot showing differentially expressed genes in COVID-19 patients 379 compared to controls. Magenta color indicates the up-regulated genes while the darkgreen 380 color represents down-regulated genes. X-axis represents fold change (log2 scale) and Y-axis 381 represent the significance level. A dashed line is drawn to indicate the significance cutoff. $\mathbf{B}$. 382 Gene-ontology analysis of up- and down-regulated genes in COVID-19 patients compared to 383 healthy subjects. Statistically significant ( $p$ value after multiple adjustments $<0.05$ ) biological 384 processes are represented. Gene members in the given processes are indicated as counts. N $385=2$ in both cases. Expression of MElightcyan (C) and MEmidnightblue (D) between COVID38619 patient and healthy control. Expression of both MElightcyan and MEmidnighhtblue modules 387 are substantially increased in patient samples and statistically significant at $P=0.06$ and 0.07 388 respectively. $\mathrm{N}=2$ each group. Unpaired t-test, two-tailed.

Fig 4. Integrative analysis of Inflammation related gene regulatory network and FDA approved drugs. A. Gene regulatory network for MElightcyan module based on proteinprotein interactions. Several therapeutic targets including insulin receptor (INSR), tumor necrosis factor (TNF), Prostaglandin-endoperoxide synthase 2 (PTGS2), Nuclear Factor Kappa B Subunit 1 (NFKB1) are present in the network. TNF and NFKB1 are two top hubs in the protein-protein interaction network of MElightcyan module. Several FDA approved drugs are available for these two therapeutic targets. Among them Pranlukast can inhibit both TNF and NFKB1. B. Vitamin D receptor (VDR), Caspase-1 (CASP1), JUN, CD55, TNFS13B, SH2B3, and Interferon Gamma Receptor 2 (IFNGR2), and IL6 are among the therapeutic targets in MEmidnightblue module. CASP1 is a key component of inflammasome and plays central role in pro-inflammatory cytokine IL-1ß production and its associated response. Antibiotic Minocycline can suppress IL-1ß production by blocking CASP1. C. In human COVID19 patients, TNF and IL-1ß are two hubs in the inflammation regulatory network. Among the inhibitors of TNF, Pranlukast acts also upon NFKB1. The antibiotic Minocycline can affect inflammasome and IL-1ß signaling and may reduce the inflammation in COVID-19. Combination of TNF inhibitor and the antibiotic Minocycline may suppress the inflammation more efficiently. List of the therapeutic targets and the available drugs are summarized in the supplementary file. 
414 Fig 5. MEbrown module represents a unique cluster associated with SARS-CoV-2 415 infection.

416 A549 cells were infected with either SARS-CoV-2, Respiratory Syncytial Virus (RSV) or 417 Influenza Virus (InV). In all conditions, A549 cells were infected at multiplicity of infection (MOI) 418 of 2 for 24 hours. A. MEbrown module shows increased expression in SARS-CoV-2 infected 419 cells compared to mock ( $p=0.0045)$. Expression of the same module is decreased in RSV ( $p$ $420=0.069)$ and $\operatorname{InV}(p=0.00012)$ infected cells. Unpaired t-tests, two-tailed. Bar plot shows 421 significant representative gene ontology terms of the MEbrown module. B. Expression of 422 MEbrown genes in COVID-19 patients. C. Integrative network analysis displays key 423 therapeutic targets and corresponding drugs in the MEbrown module. Potential therapeutic 424 targets in COVID-19 include Peptidyl Arginine Deiminase 2 (PADI2) and 5-Lipoxygenase 425 (ALOX5). One of the drugs that target ALOX5 is antibiotic Minocycline.

Fig 6. Ketogenic diet may attenuate COVID-19 induced inflammatory responses.

430 A. Heatmap displays differentially deregulated genes between influenza virus infected mice 431 either fed ketogenic diet (KD) or chow. Gene expression changes with adjusted pvalue $<0.1$ 432 were considered as differentially deregulated. B. Gene ontology of the downregulated genes 433 in KD mice compared to chow. Comparison of expression of human orthologs of (C) 434 MElightcyan, (D) MEbrown and (E) MEmidnightblue modules between chow and KD groups. 435 Changes in expression of MElightcyan and MEbrown are not significant between groups. $\mathbf{F}$. 436 Reduced expression of MEmidnightblue is noted in KD mice that is statistically significant at $P$ 437 value 0.07 . G. Human orthologs of viral response related genes are down-regulated in KD 438 group compared to chow. $\mathrm{N}=3$ each group. Unpaired t-test, two-tailed, ${ }^{*} \mathrm{p}<0.05,{ }^{* *} \mathrm{p}<0.01$.

\section{Literature}

1 Vincent, J.-L. \& Taccone, F. S. Understanding pathways to death in patients with COVID-19. The Lancet Respiratory Medicine (2020).

2 Statement on the second meeting of the International Health Regulations (2005) Emergency Committee regarding the outbreak of novel coronavirus (2019-nCoV). (World Health Organization, 2020). conformation. Science 367, 1260-1263 (2020). 
4514 Gao, Y. et al. Structure of the RNA-dependent RNA polymerase from COVID-19 virus. $452 \quad$ Science (2020).

4535 Wan, Y., Shang, J., Graham, R., Baric, R. S. \& Li, F. Receptor recognition by the novel 454 coronavirus from Wuhan: an analysis based on decade-long structural studies of SARS 455

456

457

458

6 Li, Z., Huang, Y. \& Guo, X. The brain, another potential target organ, needs early protection from SARS-CoV-2 neuroinvasion. Science China. Life Sciences, 1 (2020).

459

460 DRAFT landscape of COVID-19 candidate vaccines - 4 April 2020. (World Health Organization, 2020).

8 Beigel, J. H. et al. Advances in respiratory virus therapeutics-A meeting report from the 6th isirv Antiviral Group conference. Antiviral research (2019).

461

462

Gao, J., Tian, Z. \& Yang, X. Breakthrough: Chloroquine phosphate has shown apparent efficacy in treatment of COVID-19 associated pneumonia in clinical studies. Bioscience trends (2020).

466

467

Li, G. et al. Coronavirus infections and immune responses. Journal of medical virology 92, 424-432 (2020).

11 Hedrich, C. M. COVID-19-Considerations for the paediatric rheumatologist. Clinical Immunology, 108420 (2020).

13 Blanco-Melo, D. et al. SARS-CoV-2 launches a unique transcriptional signature from in vitro, ex vivo, and in vivo systems. bioRxiv (2020).

14 More, S. et al. Long non-coding RNA PSMB8-AS1 regulates influenza virus replication. RNA biology 16, 340-353 (2019).

15 Feldmann, M. et al. Trials of anti-tumour necrosis factor therapy for COVID-19 are urgently needed. The Lancet (2020). management of asthma. Drugs 63, 991-1019 (2003). Coomes, E. A. \& Haghbayan, H. Interleukin-6 in COVID-19: A Systematic Review and Meta-Analysis. medRxiv (2020).

19 Li, J. et al. Minocycline protects against NLRP3 inflammasome-induced inflammation and P53-associated apoptosis in early brain injury after subarachnoid hemorrhage. Molecular neurobiology 53, 2668-2678 (2016).

20 Bruno, F. et al. Recent advances in the search for novel 5-lipoxygenase inhibitors for the treatment of asthma. European journal of medicinal chemistry 153, 65-72 (2018). Heneka, M. T. et al. Neuroinflammation in Alzheimer's disease. The Lancet Neurology 14, 388-405 (2015). influenza virus infection. Science immunology 4 (2019).

23 Tomari, S. et al. Pranlukast, a cysteinyl leukotriene receptor 1 antagonist, attenuates allergen-specific tumour necrosis factor alpha production and nuclear factor kappa B nuclear translocation in peripheral blood monocytes from atopic asthmatics. Clinical \& Experimental Allergy 33, 795-801 (2003). Knowledge in Progress. Diabetes Research and Clinical Practice, 108142 (2020). 
50026 Müller, K., Ødum, N. \& Bendtzen, K. 1, 25-dihydroxyvitamin D3 selectively reduces 501 interleukin-2 levels and proliferation of human T cell lines in vitro. Immunology letters $502 \quad 35,177-182(1993)$.

50327 Pein, H. et al. Endogenous metabolites of vitamin E limit inflammation by targeting 5$504 \quad$ lipoxygenase. Nature communications 9, 1-17 (2018).

50528 Love, M., Anders, S. \& Huber, W. Differential analysis of count data-the DESeq2 $506 \quad$ package. Genome Biol 15, 10-1186 (2014).

50729 Langfelder, P. \& Horvath, S. WGCNA: an R package for weighted correlation network 508 analysis. BMC bioinformatics $\mathbf{9 ,} 559$ (2008). 

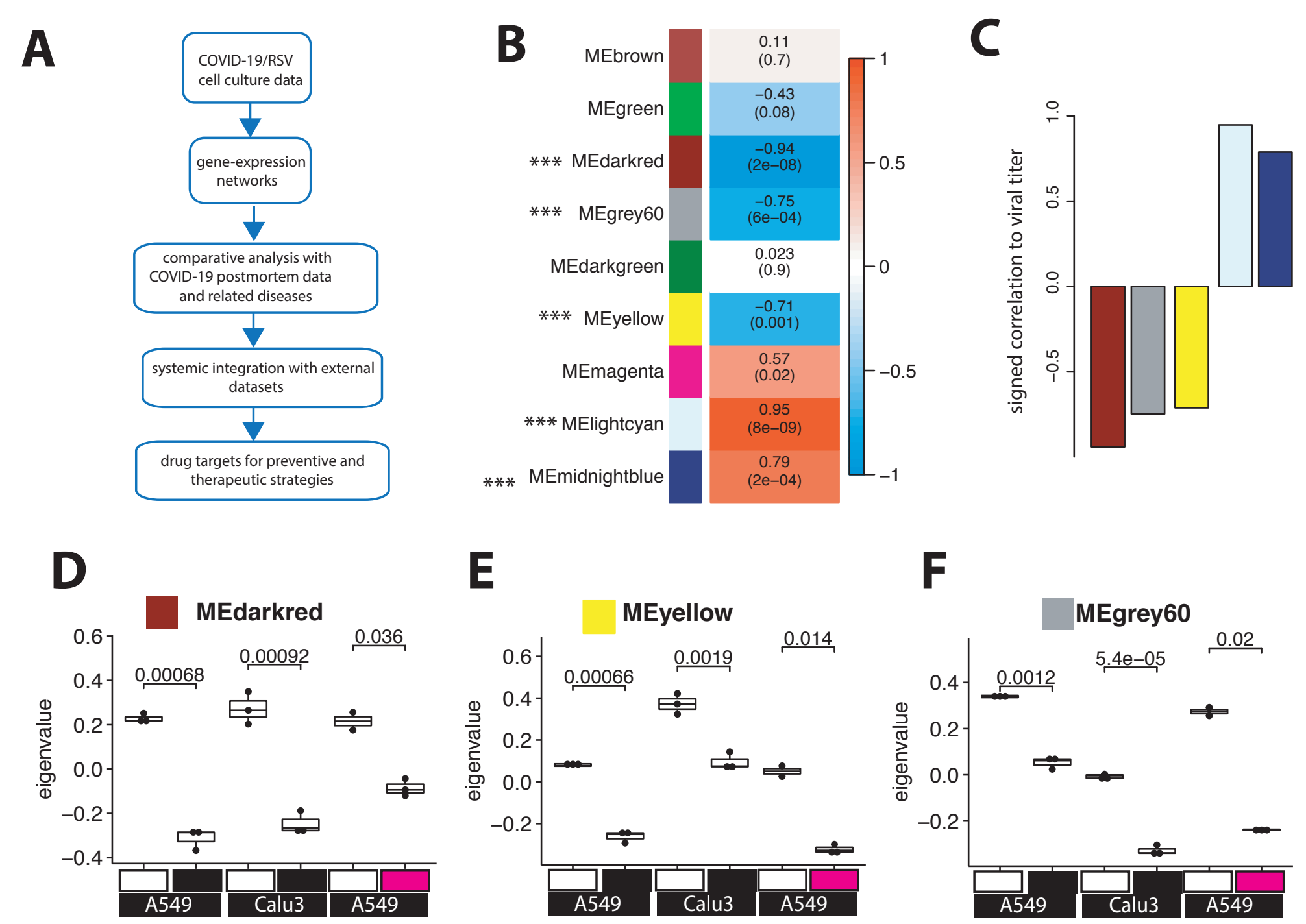

mitochondrial respiratory chain complex assembly $\mathrm{NADH}$ dehydrogenase complex assembly
cellular respiration protein folding
prollat respiration
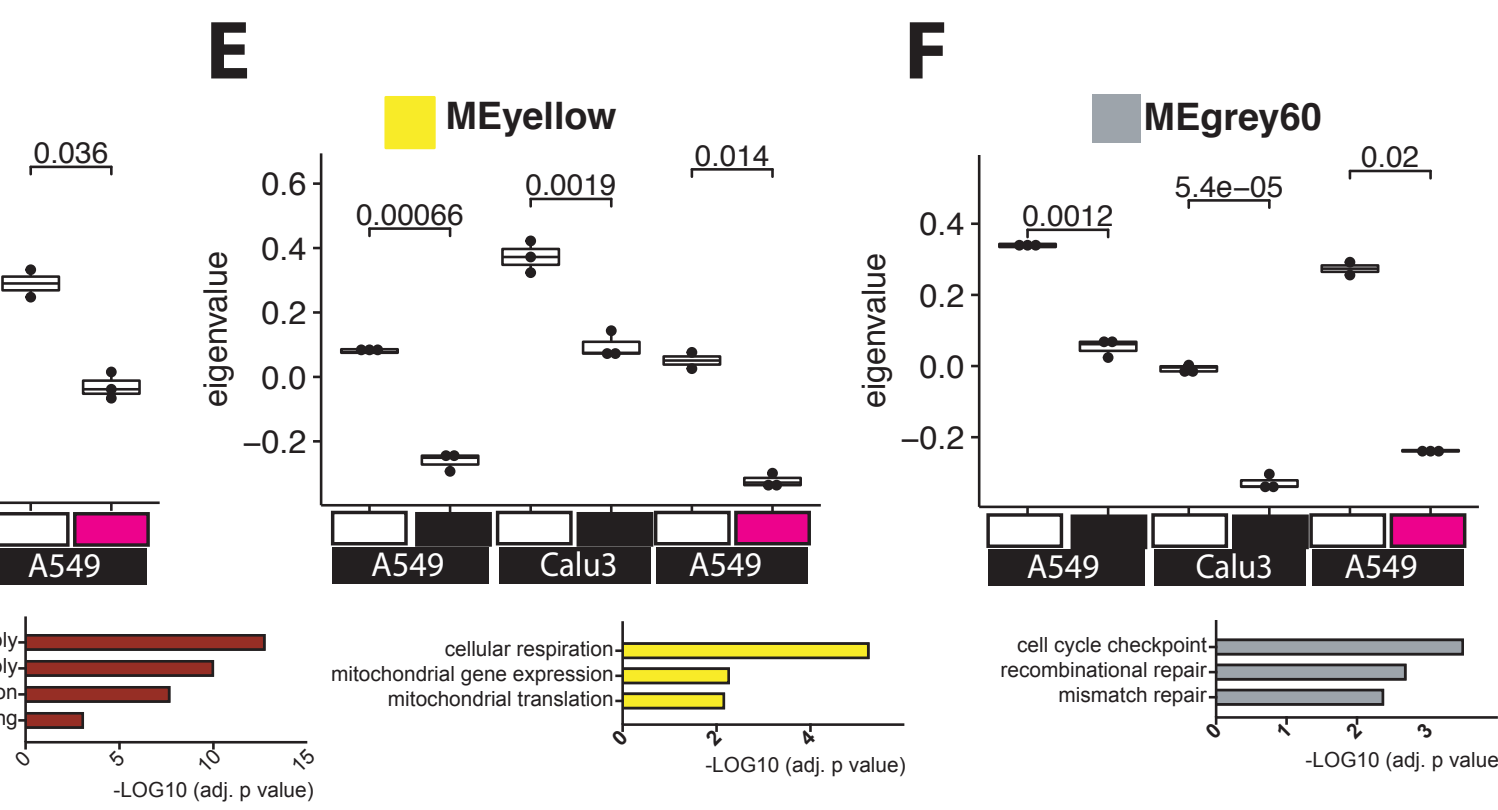

G
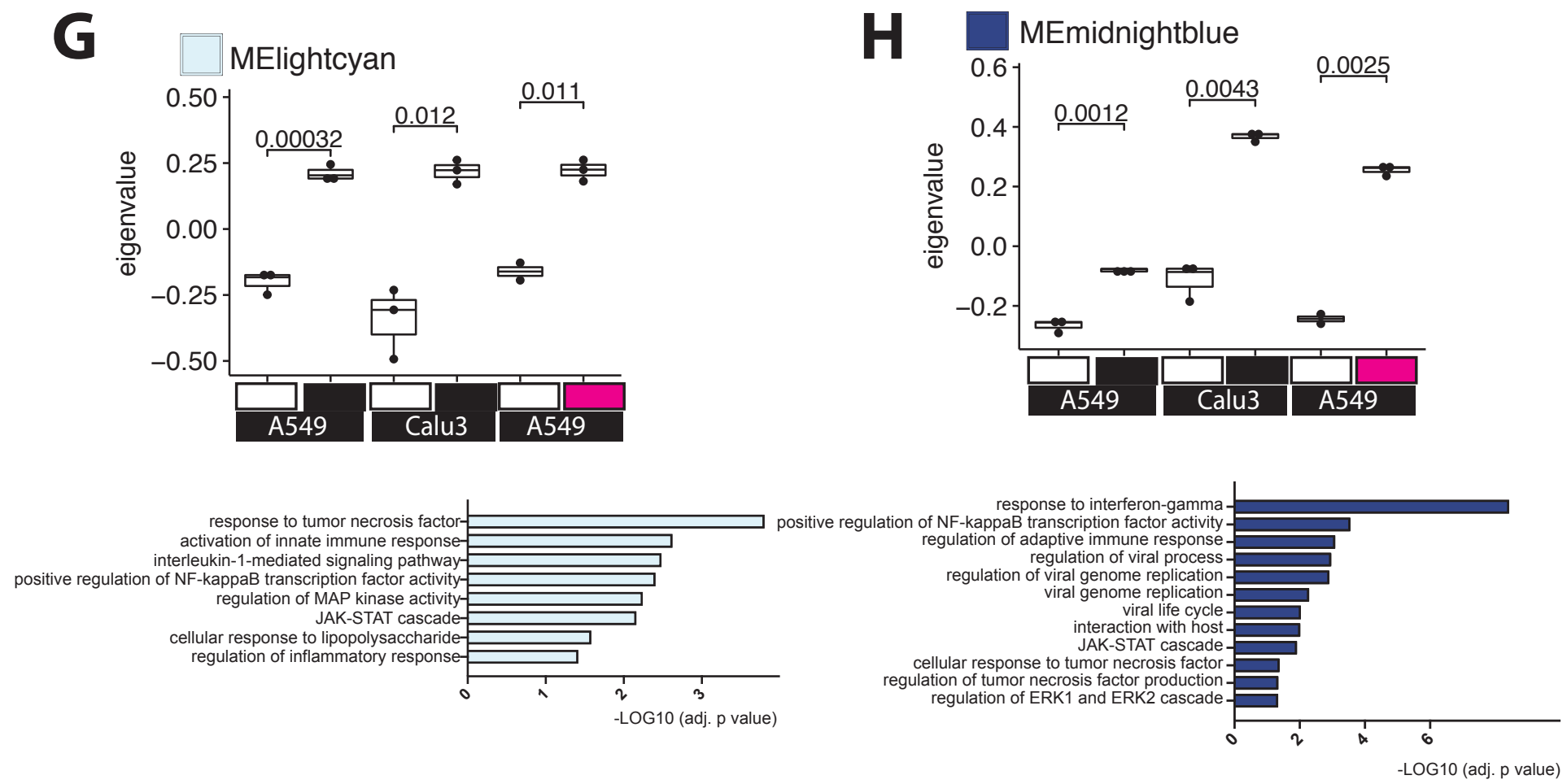


\section{A}

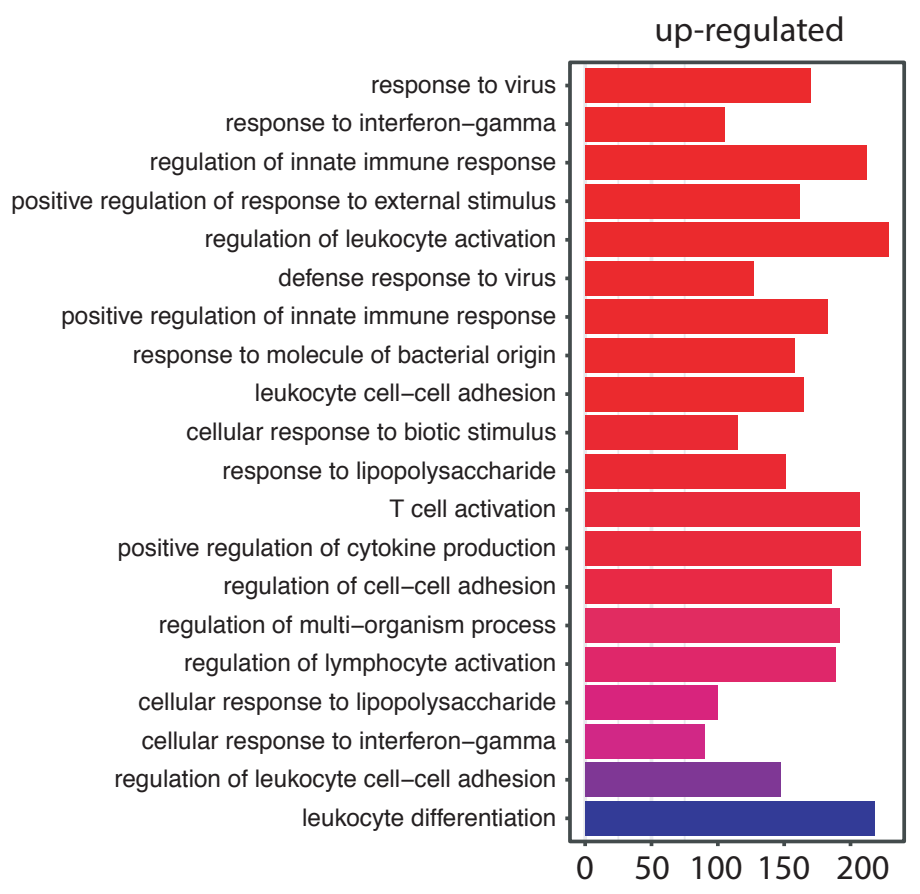

0.0000000000025

0.0000000000050

0.0000000000075

\section{B}

Lightcyan module
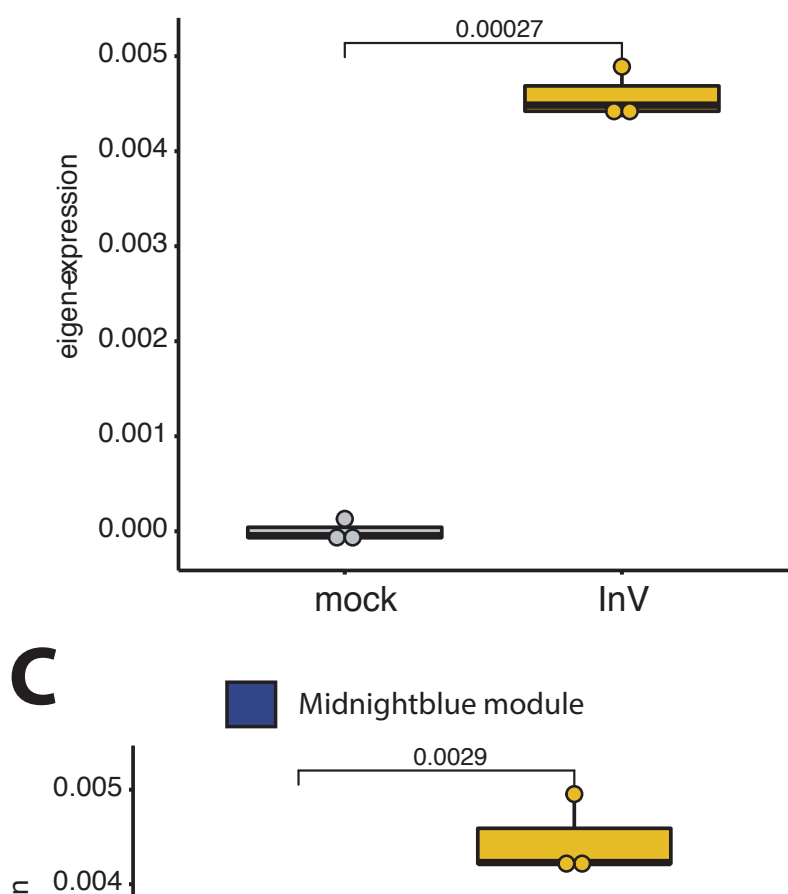

ฮำ

0.002

0.001

0.000

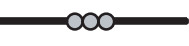

mock 
A

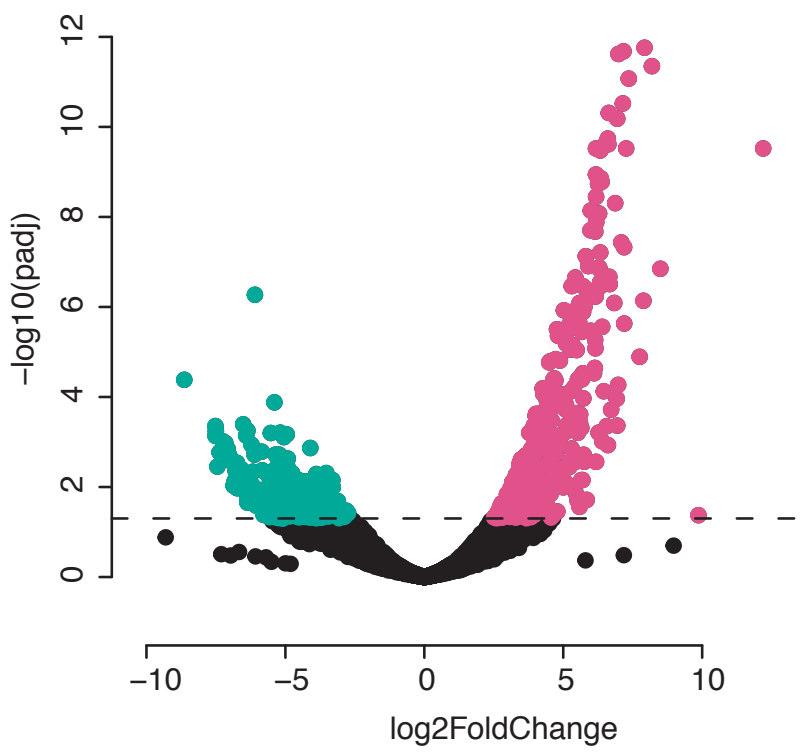

$C$

\section{MElightcyan}

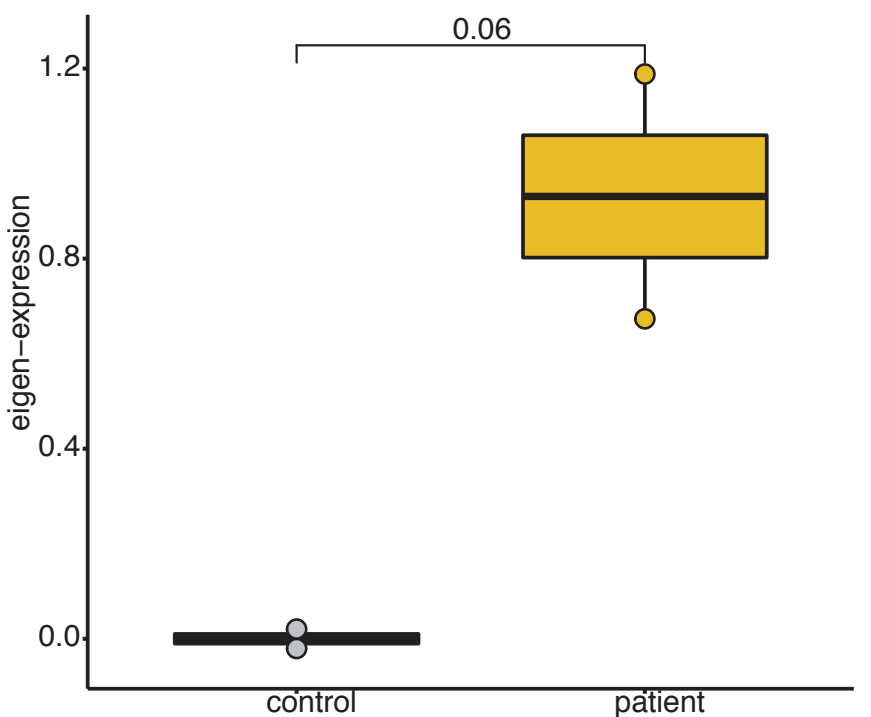

B

interaction with host viral entry into host cell. regulation of viral entry into host cell
viral genome replication.
regulation of viral genome replication. regulation of viral entry into host cell
viral genome replication.
regulation of viral genome replication. regulation of viral genome replication
viral life cycle. regulation of symbiosis through parasitism. toll-like receptor signaling pathwaytoll-like receptor 4 signaling pathway. toll-like receptor 2 signaling pathway. regulation of toll-like receptor signaling pathway response to interferon-gamma. type I interferon signaling pathway JAK-STAT cascade

\begin{tabular}{|c|}
\hline Count \\
10 \\
20 \\
30 \\
40 \\
50 \\
p.adjust \\
0.005 \\
0.010 \\
0
\end{tabular}

response to interferon-alpha neutrophil activation leukocyte migration. activation of innate immune response. response to molecule of bacterial origin $T$ cell activation. lymphocyte migration. lymphocyte mediated immunity regulation of adaptive immune response interleukin-1 secretion interleukin-1 beta secretion interleukin-6 secretion interleukin-8 secretion interleukin-12 secretion tumor necrosis factor secretion. positive regulation of MAPK cascade. ERK1 and ERK2 cascade. regulation of NIK/NF-kappaB signaling. regulation of inflammatory response. acute inflammatory response. response to oxygen levels . response to hypoxia. small GTPase mediated signal transduction response to decreased oxygen levels regulation of Ras protein signal transduction .

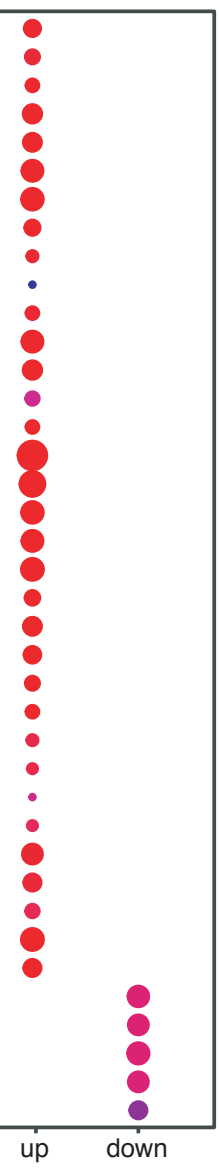

MEmidnightblue

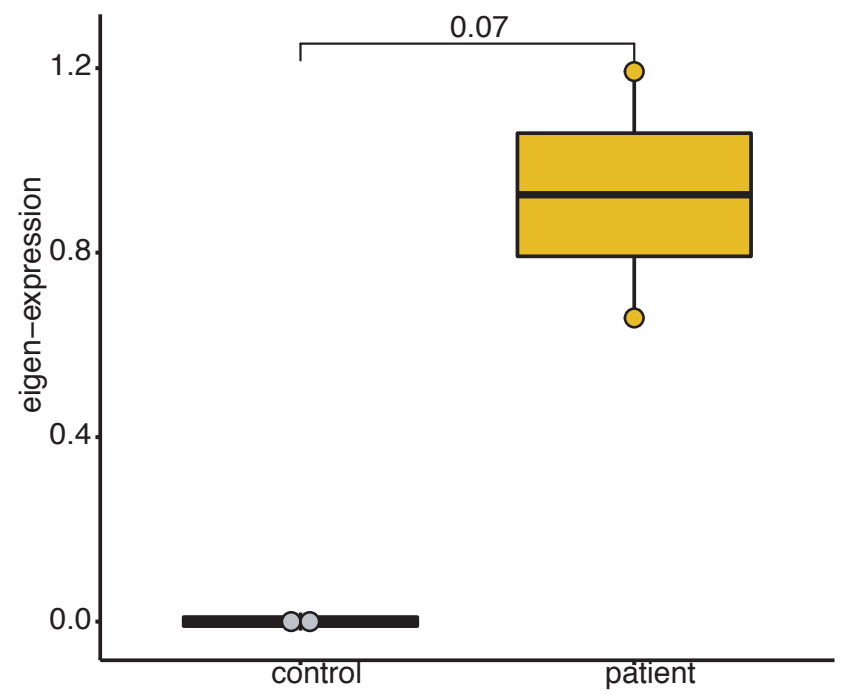




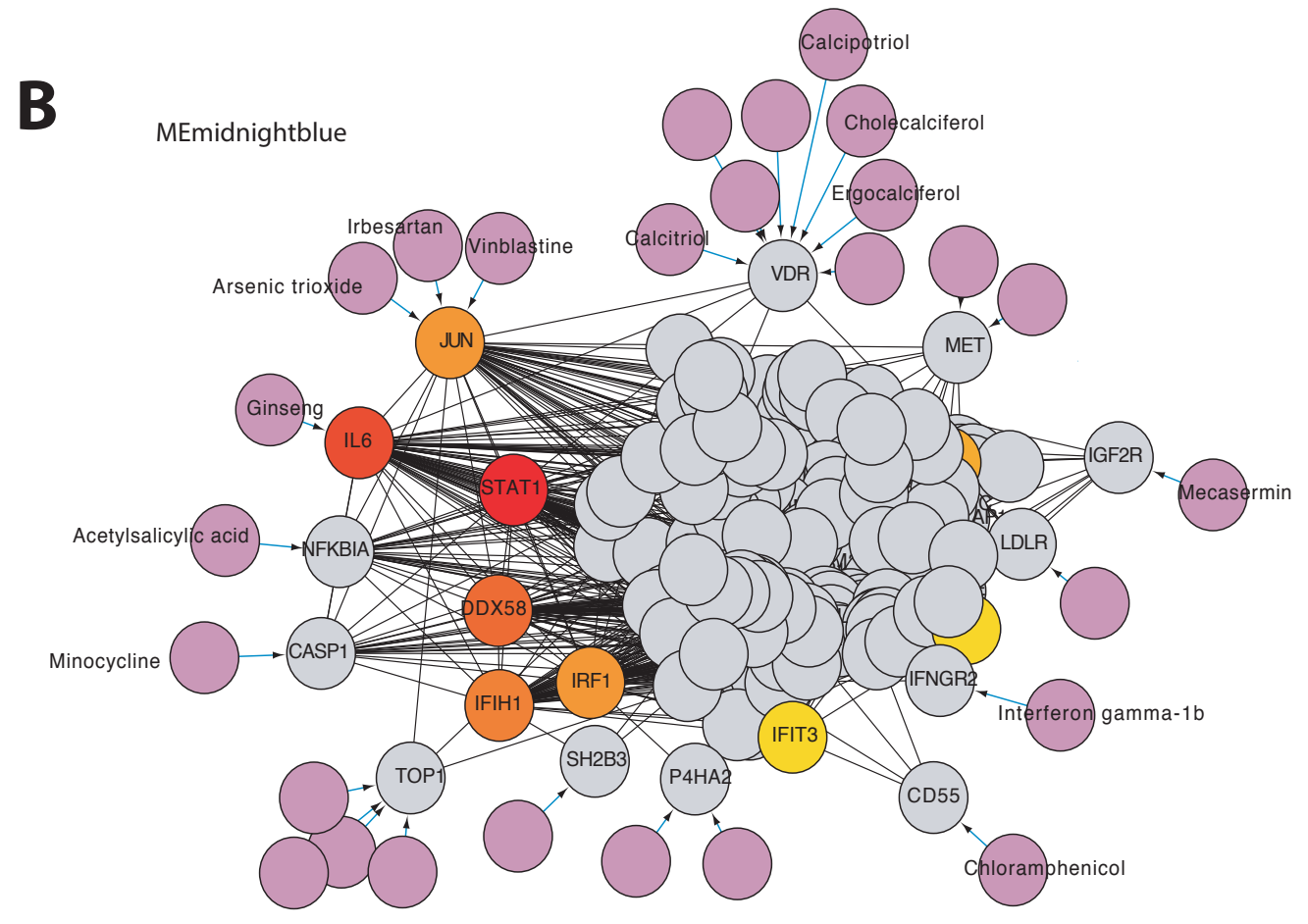

C

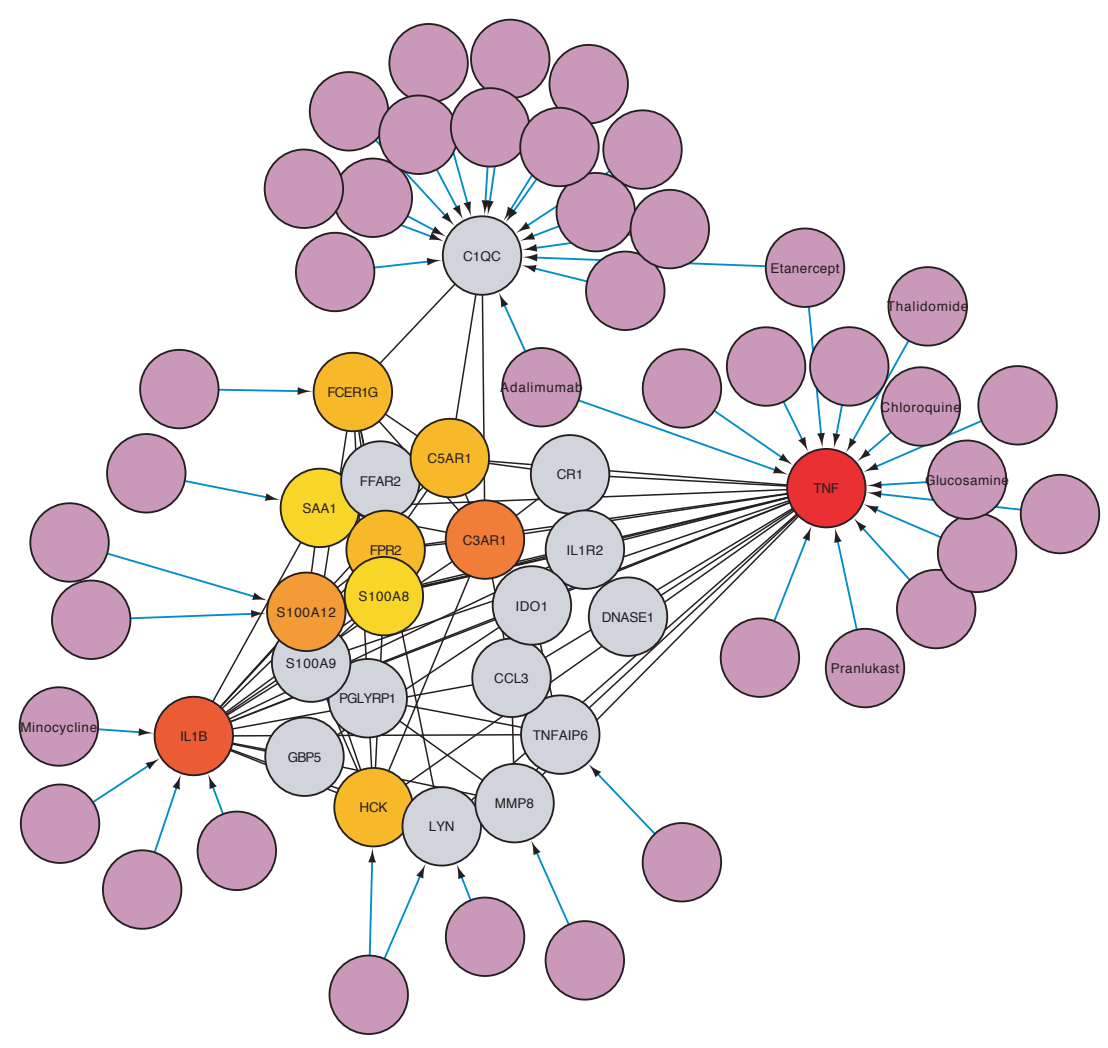


Fig. 5
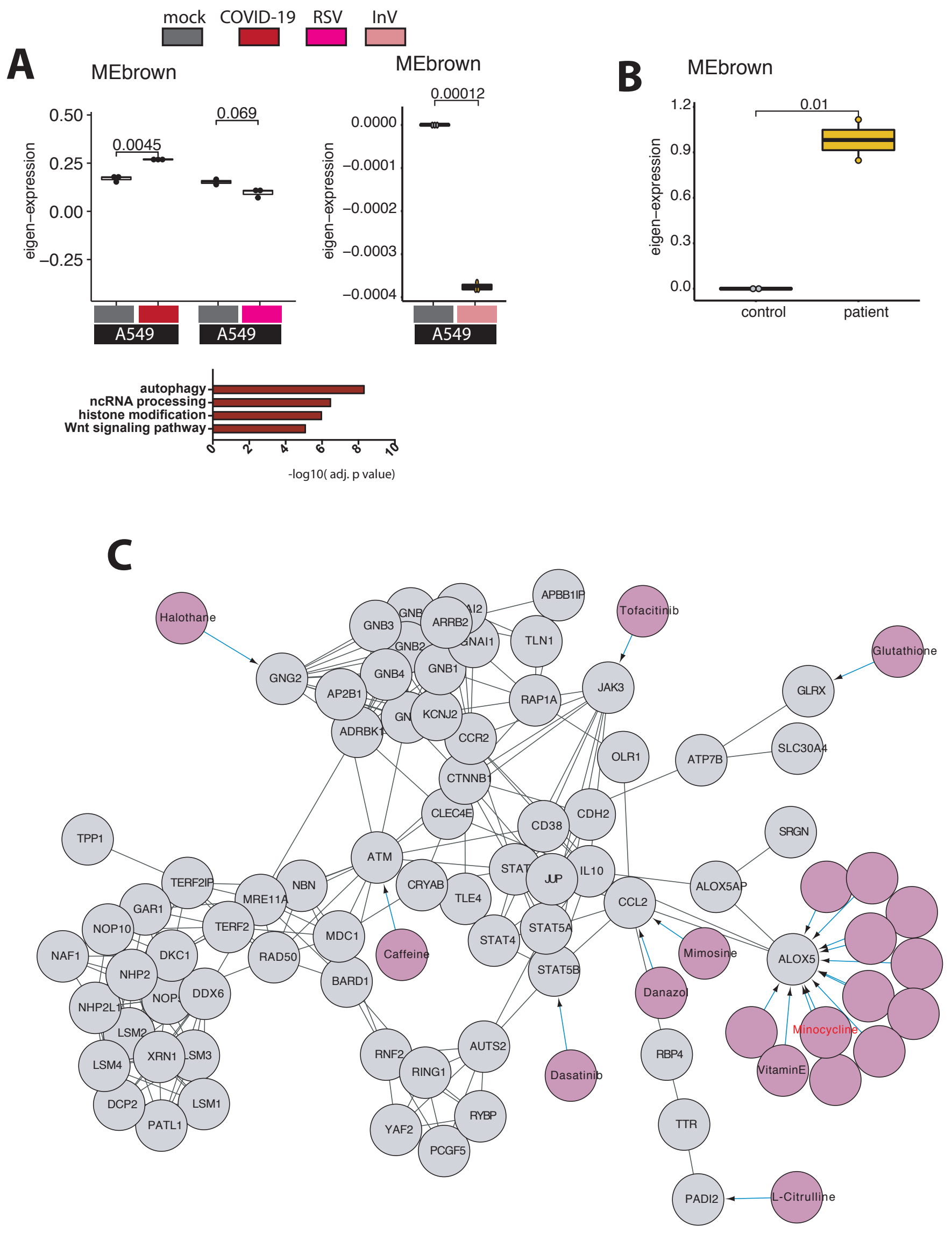

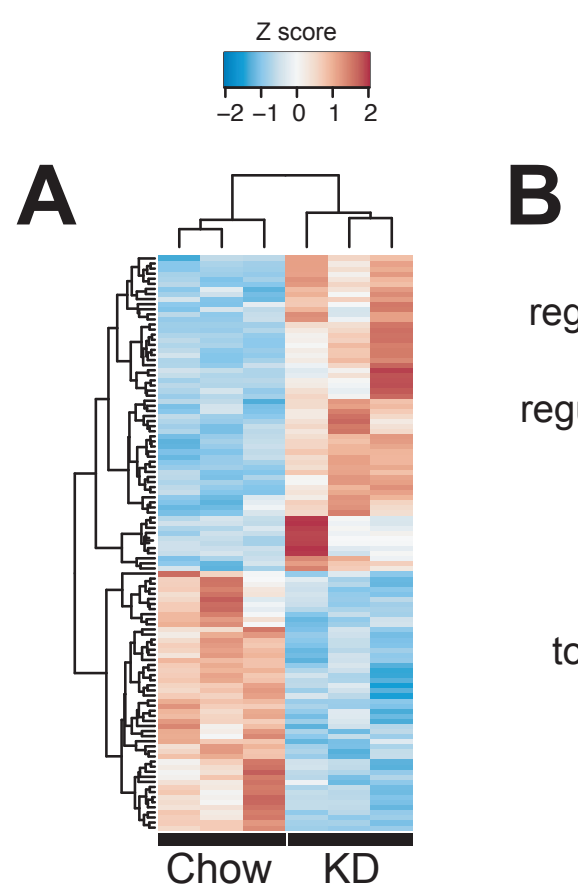

\section{downregulated}

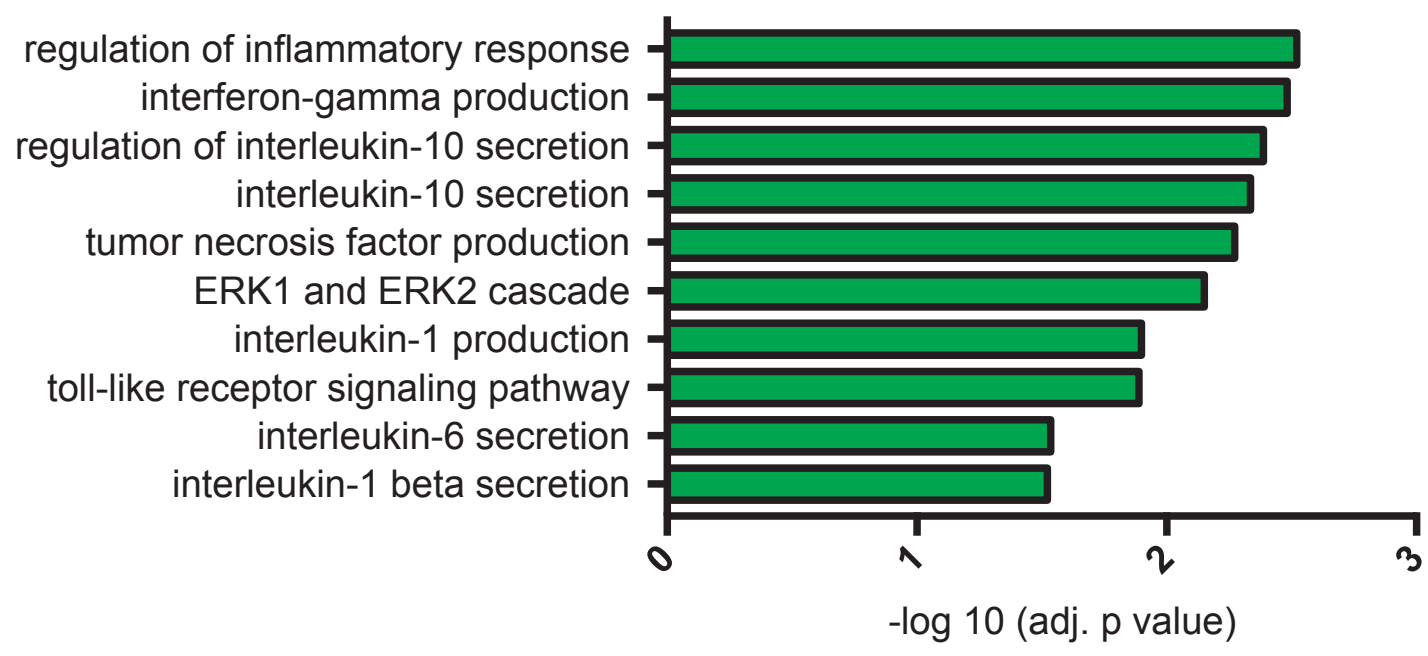

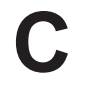

D

MEbrown
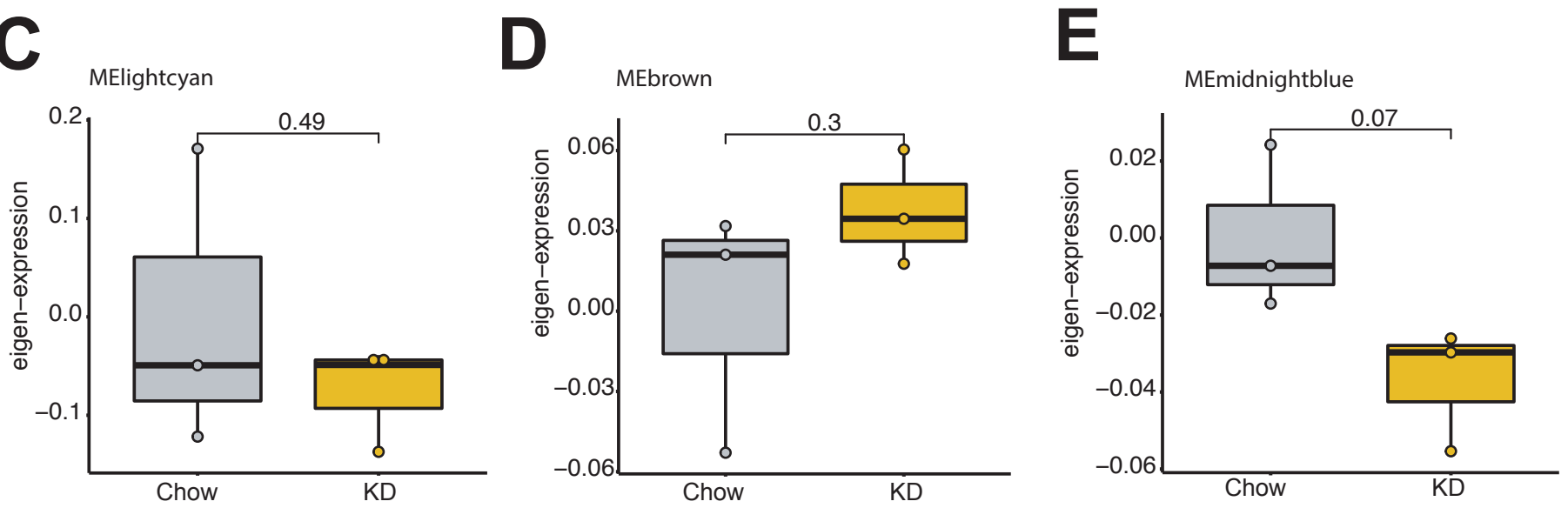

F

$\square$ normal diet + infection $\square$ ketogenic diet + infection
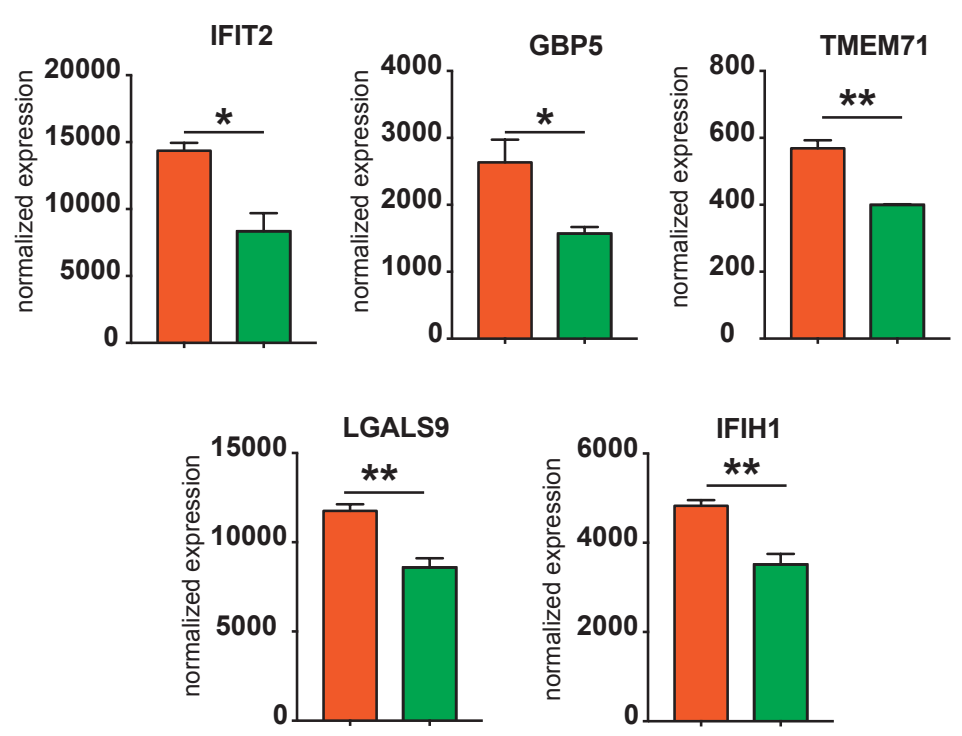